\title{
Evidence of scattering effects influenced by plasma flows in C vi $1548 \AA, 1550 \AA$ spectral lines emitted from the Sun
}

\author{
C. Gontikakis ${ }^{1}$ and J.-C. Vial ${ }^{2,3}$ \\ 1 Research Center for Astronomy and Applied Mathematics, Academy of Athens, 4 Soranou Efessiou Str., 11527 Athens, Greece \\ e-mail: cgontik@academyofathens.gr \\ 2 Université Paris Sud, Institut d'Astrophysique Spatiale, UMR 8617, 91405 Orsay, France \\ 3 CNRS, Institut d'Astrophysique Spatiale, UMR 8617, 91405 Orsay, France
}

Received 12 January 2016/ Accepted 12 April 2016

\begin{abstract}
Aims. We search for, and study, individual spectral profiles where the complex shape of the C IV $1548 \AA$ line is different from the shape of the simultaneously recorded C IV $1550 \AA$ line. Such an asymmetry is not expected for line emission resulting from collisional excitation. Therefore we propose an explanation of these observations through the differential effect of velocity fields on resonant scattering.

Methods. We analyse spectra recorded with the Solar Ultraviolet Measurements of Emitted Radiation (SUMER) on the Solar and Heliospheric Observatory (SOHO) over active region, NOAA 8541 as well as a second data set on the quiet Sun. We perform Gaussian fits on the individual profiles with two or three Gaussian functions. Moreover, we parameterize the profile asymmetries by calculating the intensity ratios $I_{1548} / I_{1550}$, from the derived Gaussian functions. We also calculate artificial spectral profiles emitted from two plasma volumes, which have different line of sight motions and where the plasma emission is influenced by resonant scattering.

Results. We locate three small regions in NOAA 8541 which have spectral asymmetries. There the profiles have two or three spectral components, with different intensity ratios. Artificial profiles show that two plasma volumes, having distinct velocities relative to the observer, may reproduce the observed profiles, under the influence of resonant scattering.

Conclusions. Asymmetric profiles, found in an active region, can be used as a diagnostic for the importance of resonant scattering in transition region plasma.
\end{abstract}

Key words. Sun: transition region - Sun: UV radiation

\section{Introduction}

The dominant mechanism of light emission from the solar transition region and coronal plasma, mostly in the quiet Sun, is the collisional excitation followed by spontaneous radiative decay (Mariska 1992). In the extended solar corona, observed by coronagraphs, radiative scattering dominates over collisional excitation because of the latter's weakness caused by the low electron density. There, the intensity ratio of lines doublets, such as the O VI $1032 \AA$ and $1038 \AA$ formed through radiative scattering, can be used to measure the solar wind velocity (Kohl \& Withbroe 1982; Noci et al. 1987). In active regions and also in impulsive events, such as flares, bright points, or jets, the resonance scattering of the radiation coming from these bright regions has a chance to compete with thermal emission (Judge \& Pietarila 2004). Gontikakis et al. (2013) showed that in active region NOAA 8541 , the C IV $1548 \AA$ and $1550 \AA$ spectral lines' emission was caused by resonant scattering, in the vicinity of a microflare.

The C IV $1548.202 \AA$ and $1550.774 \AA$ (see Teriaca et al. (1999) and the National Institute of Standards and Technology (NIST) atomic data base, Kramida et al. (2014), for the exact wavelengths) are important spectral lines for the study of the solar transition region. The $\mathrm{C}^{3+}$ ion, which is a lithiumlike ion, has a maximum concentration at the temperature of $100000 \mathrm{~K}$ (Arnaud \& Rothenflug 1985) which is typical for the transition region. Moreover, C IV $1548 \AA$ (where we truncate the wavelength decimals for simplicity) is a bright spectral line, where accurate Doppler measurements can be performed. The C IV $1548 \AA$ line is part of a doublet with the C IV $1550 \AA$ line. The $1550 \AA$ line has half the intensity of $1548 \AA$ when emitted by an optically thin plasma. The two spectral lines are emitted by the same ion, which means by the same plasma. Therefore, one expects that study of the two spectral lines should provide the same Doppler shifts, for example, as long as systematics errors (such as blends) can be corrected.

However, there are cases in the literature where the C IV $1548 \AA$ and $1550 \AA$ spectral lines are significantly different in their Doppler shifts, something mentioned but not explained by the authors. In Lites \& Cook (1979), the C IV $1548 \AA$ and $1550 \AA$ lines emitted by a small flare, recorded with the Skylab/S082B slit spectrograph present different spectral shapes. The C IV $1548 \AA$ and $1550 \AA$ lines emitted by the $\beta$ Dra star (Wood et al. 1997) have also different shapes (see their Fig. 9). In the above mentioned cases, the total intensity ratio of the $1548 \AA$ line over the $1550 \AA$ line is smaller than two which indicates that the plasma is optically thick (Dere \& Mason 1993).

Moreover, in studies of the average Doppler shifts of transition region lines relative to the chromosphere, performed with the Solar Ultraviolet Measurements of Emitted Radiation (SUMER) spectrograph (Wilhelm et al. 1995), measurements of the C IV $1548 \AA$ and $1550 \AA$ lines differ in the quiet Sun by an amount of $5 \mathrm{~km} \mathrm{~s}^{-1}$ which is larger than the uncertainties 
Table 1. SUMER observations analysed and used in this work

\begin{tabular}{ccccc}
\hline \hline Number & Date & Time (UT) & $\begin{array}{c}\text { Pointing } \\
x\left({ }^{\prime \prime}\right), y\left({ }^{\prime \prime}\right)\end{array}$ & $\begin{array}{c}\text { Field of } \\
\text { view (") }\end{array}$ \\
\hline 1 & $15-05-99$ & $12: 58-13: 00$ & 331,384 & $1 \times 120$ \\
2 & $15-05-99$ & $13: 00-13: 01$ & 331,384 & $1 \times 120$ \\
3 & $15-05-99$ & $13: 02-13: 04$ & 331,384 & $1 \times 120$ \\
4 & $15-05-99$ & $13: 04-13: 57$ & 331,384 & $159 \times 120$ \\
5 & $15-05-99$ & $13: 58-14: 53$ & 331,384 & $159 \times 120$ \\
\hline
\end{tabular}

(Teriaca et al. 1999). In other examples, such as in Brekke et al. (1997) the Doppler shift variation of the two C IV lines is of $2 \mathrm{~km} \mathrm{~s}^{-1}$, this time smaller than the uncertainty.

In the following study we examine similar effects that can be explained by the mechanism of resonant scattering.

In Sect. 2 we present the observed data, from active region NOAA 8451 as well as from other locations, the data treatment, and a summary of the data analysis. In Sect. 3, we show asymmetric individual spectral profiles where the C IV $1548 \AA$ has a different spectral shape from the C IV $1550 \AA$ even if both profiles are observed at the same location and at the same time. In Sect. 4 we present a statistical analysis of the NOAA $8451 \mathrm{C}$ IV $1548 \AA$ and $1550 \AA$ spectra in an effort to recognise the asymmetric spectra from the ordinary spectra. In Sect. 5 we present quantitatively the two main effects, the collisional excitation and the radiation scattering, that affect the formation of the two C IV lines. We also present a simple scenario of the differential effect of velocity on resonant scattering which may explain the observed asymmetric profiles. Finally, for several spectral parameters, we compute artificial profiles of the C IV spectral lines, that are similar to the observed ones. In Sect. 6, we discuss our results and present our conclusions.

\section{Observations}

We revisited a number of data sets, where SUMER simultaneously recorded the C IV $1548.202 \AA$ and $1550.774 \AA$ spectral lines, at the same locations on the Sun. The characteristics of these data sets are detailed in Table 1. The data from 15 May 1999 are recorded on active region NOAA 8541 (Winebarger et al. 2002; Gontikakis et al. 2013). On 15 May, SUMER performed two rasters that recorded five spectral lines: Ne VIII $770 \AA$, (in second order), Si II $1533 \AA$, C IV $1548 \AA$ and $1550 \AA$ and a section of the continuum at $1528 \AA$. The spectral lines were recorded in spectral windows about $2 \AA$ wide (about $1 \AA$ for the second order line). The other observations are full detector readouts of spectra with a spectral range from $1526 \AA$ up to $1560 \AA$. We also studied data from a full detector readout recorded over a quiet Sun area on 23 February 1996 (Teriaca et al. 1999). From this exposure we analysed a spectrum with a wavelength range from $1155.39 \AA$ up to $1594.96 \AA$.

We applied the standard corrections available from the solar-soft library for the SUMER data including the dead time correction, the application of the flatfield, the correction of the local gain and the geometric distortion corrections. We also transformed the intensity values in physical units using the "Radiometry" routine.

The dispersion axis of the spectra recorded during the raster on NOAA 8541 , has been calibrated using the single exposure data sets in the lines 1 to 3 of Table 1 (see Gontikakis et al. 2013).
We studied these data to find instances where the C IV $1548 \AA$ spectral line has a different spectral shape from the C IV $1550 \AA$ line, and the two lines are recorded simultaneously and on the same spatial location. We call these cases asymmetric profiles.

We examined various parameters of the spectral profiles, either computed using Gaussian fits, such as the spectral line position, and the line width, or parameters such as the skewness of the profiles. We compared the computed parameters from the two C IV spectral lines to see if we could quantify the shape variations of the two profiles. Moreover we wanted to check if the profile variations depended on some specific observed quantity. We will present in detail these results in Sect. 4.

We also visually selected individual profiles of the two C IV lines $1548 \AA$ and $1550 \AA$, where the two lines have a notably different spectral shape. We took care to avoid profiles with count rates higher than, or of the order of, 50 counts/s/pixel because such profiles have complex spectral features caused by the saturation of the detector (see Gontikakis et al. 2013). A good way to track such unusual events in the two rasters is to compute a map of the spectral line width using a single Gaussian fit. In this map the candidate features appear clearly because of the larger spectral width, even if the single Gaussian fit is a poor description of the true spectral profile. We found 14 individual spectral profiles, with such asymmetries, all in data from the NOAA 8541 active region (see Fig. 2) at three distinct spatial regions. Region 1 was recorded by SUMER at 13:52 UT, during the first raster, at $(x, y)=\left(390^{\prime \prime}, 348^{\prime \prime}\right)$. Region 2 at 14:52 UT at $(x, y)=\left(257^{\prime \prime}, 368^{\prime \prime}\right)$, and region 3 at 14:39 UT at $(x, y)=\left(294^{\prime \prime}, 405^{\prime \prime}\right)$. Regions 2 and 3 were recorded during the second raster. The size of region 1 is $3.5^{\prime \prime} \times 5^{\prime \prime}$ while Regions 2 and 3 are of $1^{\prime \prime} \times 4^{\prime \prime}$ and $1^{\prime \prime} \times 2^{\prime \prime}$ respectively. The selected profiles were complex, and a single Gaussian fit could not describe them. In one location, the C IV $1548 \AA$ and $1550 \AA$ profiles are described with three Gaussian components. These individual profiles are described in the next section.

We also used Transition Region And Coronal Explorer (TRACE) images recorded on 15 May 1999. We co-aligned the $1216 \AA$ image, recorded on 14:16:06 UT, with the Si II $1533 \AA$ image of the second SUMER raster. For the co-alignment we used image correlation and visual inspection. There were no simultaneous TRACE images with any of the three events.

\section{Observed spectra showing differences between the two $C$ IV lines}

In Fig. 1 we see 6 different spectral profiles of the two C IV lines taken from the three different regions 1,2 and 3 of NOAA 8541. Panels a to $d$ are recorded over Region 1, and panels e and $f$ are recorded over Regions 2 and 3 respectively. The two vertical lines in each panel of Fig. 1 correspond to the rest wavelength of the two C IV spectral lines. We present the two C IV spectral lines on the same wavelength axis. The wavelength band between $1548.9 \AA$ and $1549.4 \AA$ was not recorded by SUMER. The small wavelength size of the spectral windows caused a truncation at the long wavelengths of both C IV spectral lines due to the large line-width of these individual profiles.

We performed Gaussian fits on the profiles with two or three Gaussian functions depending on the case. We always used the same number of Gaussians for the two lines in a pair. Moreover, we added constraints on the Gaussian fit, such that the spectral components of the C IV $1548 \AA$ line have the same Doppler shift and Doppler width, as the corresponding spectral components 

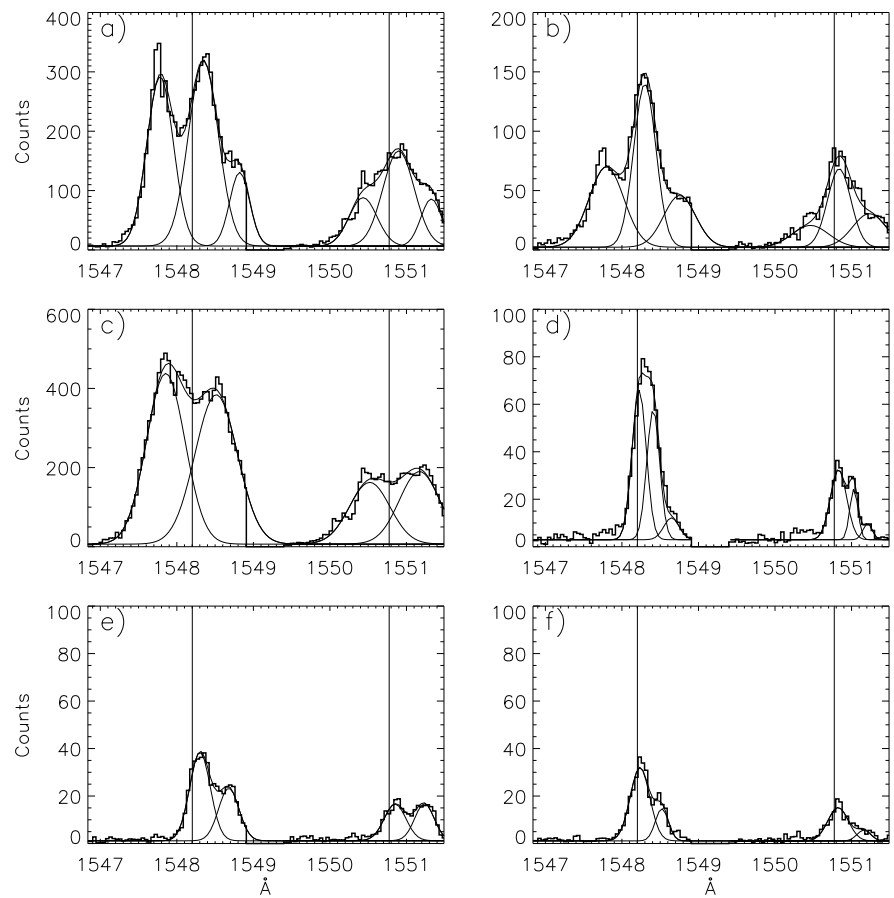

Fig. 1. Individual profiles where the spectral shape of the C IV $1548 \AA$ is different from the shape of the co-local and simultaneously recorded C IV $1550 \AA$ line. The vertical lines show the zero velocity wavelength of the $1548 \AA$ and $1550 \AA$ spectral lines. The interval between $1548.9 \AA$ and $1549.4 \AA$ is due to the too-short spectral windows used during the rasters. Panels a) to d) are recorded over Region 1 while panels e) and f) over Regions 2 and 3 respectively. Data points are represented with a thick histogram line. The thin lines represent the Gaussian functions needed to fit the data.

of the C IV $1550 \AA$ line. For panels a, b and d we used three Gaussian functions, and two for panels c, e and f. For the fit of the C IV $1548 \AA$ line, in Figs. 1a and b, we also included three data points of the C IV $1550 \AA$ spectral window, between $1549.5 \AA$ and $1549.7 \AA$ in order to better constrain the fit.

In panels a and $\mathrm{b}$, the first spectral component is blueshifted while the two other components are redshifted. The Gaussian functions in panels $a$ and $b$, representing the most redshifted spectral components of C IV $1548 \AA$, extrapolate the nonrecorded spectral profiles further than the red edge of the $1548 \AA$ spectral window. The same is true for the redshifted Gaussians in panels $\mathrm{c}$, e and $\mathrm{f}$.

The profiles in panels a to $c$ are mostly characterised by the presence of a spectral peak in the blue wing of the C IV $1548 \AA$ line, at $1547.75 \AA$. This spectral peak position, corresponds to a Doppler velocity of $86 \mathrm{~km} \mathrm{~s}^{-1}$. The same Doppler position for the C IV $1550 \AA$ line is at $1550.5 \AA$. There, the profile shows a spectral peak which is much weaker than that of C IV $1548 \AA$. In panel c, the third, most redshifted component, present in panels a and $\mathrm{b}$ disappears.

The $1550 \AA$ profile in panel $\mathrm{d}$ has a peculiar self-reversal which is absent from the $1548 \AA$ line. There are two more profiles, in Region 1, with the same shape in adjacent spectral pixels. Therefore, we excluded the possible explanation that this effect is due to counting statistics. We applied a fit with three Gaussian functions to these peculiar profiles. The Doppler shift and width of the fits on the C IV $1548 \AA$ line were constrained from the fits on the C IV $1550 \AA$ line. In this approach, the reversed profile is explained as two redshifted main spectral components. The third spectral component, in the C IV $1548 \AA$ and $1550 \AA$ lines seem to correspond to spectral blends: Si I $1548.72 \AA$ and $1551.24 \AA$ respectively for the two lines. However, the Gaussian functions of these blend lines have a large non-thermal velocity of $19 \mathrm{~km} \mathrm{~s}^{-1}$ and $11 \mathrm{~km} \mathrm{~s}^{-1}$ which does not correspond to chromospheric lines. In the other two reversed profiles, the third component has a low intensity and a high line width.

Of course the self-reversal in the C IV $1550 \AA$ line could correspond to opacity. In this case the multi Gaussian fit is useless. However, the C IV $1548 \AA$ has twice the optical thickness of the C IV $1550 \AA$ line and one should expect a higher self reversal for the $1548 \AA$ line.

Finally, in panels e and f, from Regions 2 and 3 respectively, the individual profiles have two spectral components. In panel e, for the C IV $1548 \AA$ profile, the spectral peak at $\simeq 1548.3 \AA$ has 1.6 times higher intensity than the peak at $1548.7 \AA$. On the other hand, the two spectral peaks of the C IV $1550 \AA$ at $\simeq 1550.9 \AA$ and $\simeq 1551.2 \AA$ have roughly the same intensity. The same features can be seen in panel $\mathrm{f}$. Using single Gaussian fits to the C IV $1550 \AA$ line from the three regions, we calculated the non-thermal velocities. The C IV $1550 \AA$ A non-thermal velocities in Fig. $1 \mathrm{~b}$ to $\mathrm{d}$ are $116 \mathrm{~km} \mathrm{~s}^{-1}, 75 \mathrm{~km} \mathrm{~s}^{-1}$, and $40 \mathrm{~km} \mathrm{~s}^{-1}$ respectively.

In Fig. 2 we can see the spatial location of the three small regions from which we selected the spectra of Fig. 1. In Figs. 2a and b we see the intensity images of C IV $1548 \AA$ line taken during the two SUMER rasters of NOAA 8541 . The three regions of interest are indicated with arrows. Region 1 recorded on 13:52 UT during the first raster is seen in panel a. Regions 2 and 3 recorded on 14:52 UT and 14:39 UT respectively during the second raster are seen in panel b. Only Region 1 is apparent as a bright region in intensity in C IV $1548 \AA$ (see Fig. 2a) and in Si II $1533 \AA$ (panel e), while all of them appear to have large line widths in Figs. $2 \mathrm{c}$ and d. Figure $2 \mathrm{f}$ shows the TRACE images in the $1216 \AA$. Region 1 is in the vicinity of two sunspots (see Figs. 2e, f) while Regions 2 and 3 are inside plage areas (see Figs. 2e and f).

The three features, especially that in Region 1, are reminiscent of explosive events (Dere et al. 1989). Explosive events belong to a large category of solar phenomena that has a size of a few arcseconds, with a duration of one to three minutes. Their emission lines show complex profiles with high Doppler shifts. Explosive events may be explained as jets emanating from a magnetic reconnection site (Innes et al. 2015) or even as a rotational plasma motion around the axis of a spicule-like feature (Curdt \& Tian 2011).

\section{Results of statistical analysis}

From our datasets, we computed the moments of all the individual spectral profiles of C IV $1548 \AA$ and $1550 \AA$, in order to build statistics on their properties. Our basic purpose was to check if one can distinguish the asymmetric profiles. We computed the profiles' Doppler shifts and Doppler widths using the first and second moments, respectively, rather than using the Gaussian fit parameters. The reason for this is that the asymmetric profiles do not have a single Gaussian shape. We also computed the skewness, using the third moment, of the profiles. In the skewness calculation, as the profile's far wings are excessively weighted, with a weight factor equal to the cube of the wavelength, we truncated the profiles at a few spectral pixels from the profile's 

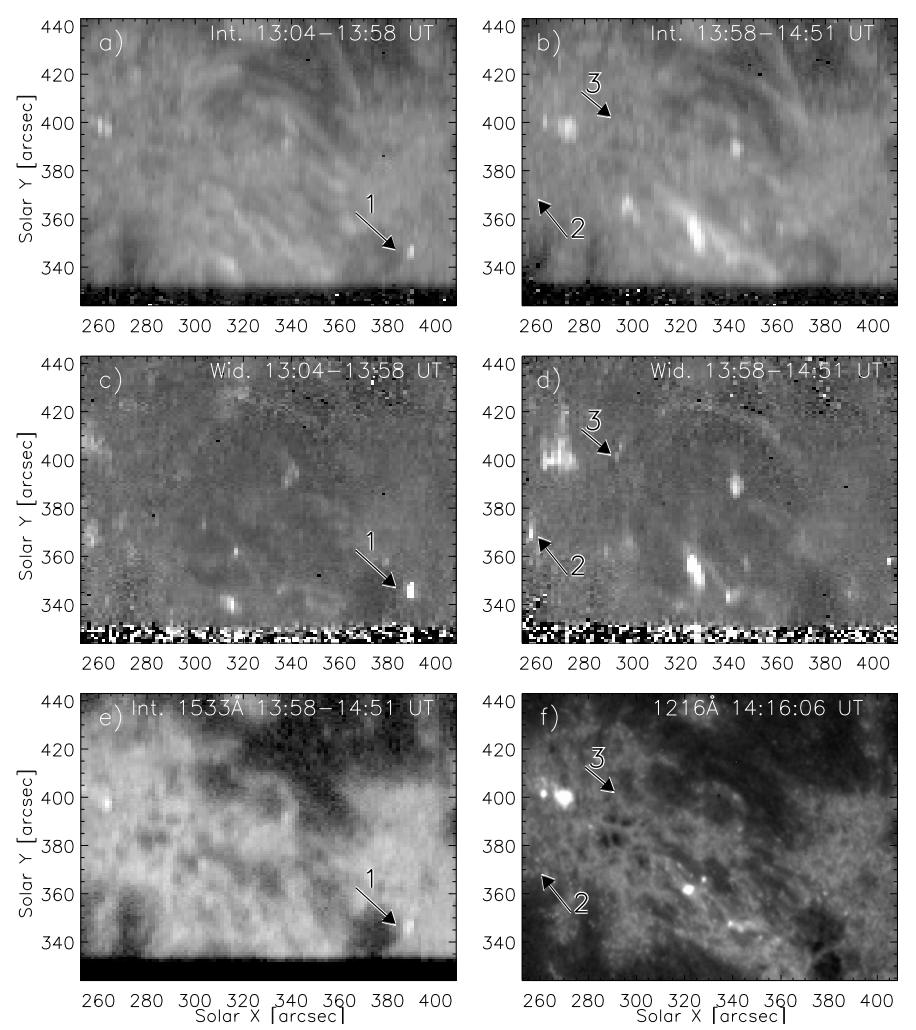

Fig. 2. Images of AR 8541, recorded by SUMER in C IV $1548 \AA$ (panels a) to d)), and in Si II $1533 \AA$ (panel e)) and TRACE (panel f)) where the location of three regions showing individual asymmetric profiles are indicated with arrows. The first row shows the intensity image of the two SUMER rasters while the second row, the line width map. The third row shows the Si II $1533 \AA$ and the $1216 \AA$ images recorded with SUMER and TRACE respectively. Notice that the regions appear to have a high line width (panels c) and d)) while only Region 1 appears as a high intensity area (panels a) and e)).

central position. The best results are obtained for a truncation at \pm 6 spectral pixels from the mean profile position, even if this truncation left out a significant part of the profiles, which have a high line width (see Fig. 1). In Fig. 3 we show the scatter plots of the Doppler shift, the Doppler width and the skewness of the profiles. Figure 3 shows values only for the profiles where the total intensity, this time computed with a Gaussian fit, is higher than 290 counts to reduce noise in the data. In panels a and b, the shift of the profiles is defined using their relative positions within the 50 pixel size spectral windows. The spectral pixels correspond to $\simeq 0.04 \AA$ (Curdt et al. 2001). The most successful identification of asymmetric profiles is obtained for the Doppler shift scatter plot of Fig. $3 \mathrm{~b}$ for the points of Region 2 which are all in the tail of the distribution of points. However this is not the case for the two points of Region 3 that are located in the core of the distribution. Moreover, in Fig. 3b other points lay outside the core of the distribution which, on visual inspection do not show any asymmetry. The same behaviour is obtained from the other scatter plots where part of the values computed from the asymmetric profiles are at the tail of the distributions while the rest of them are in the core of the distributions. Curiously, in Fig. 3d there is no clear distinction between the asymmetric and non asymmetric points. In Figs. 3e and f, the skewness also can indicate the presence of asymmetric profiles even if the calculation of this moment suffers from the presence of noise. We used this statistical study for tracking asymmetric profiles in our data set,
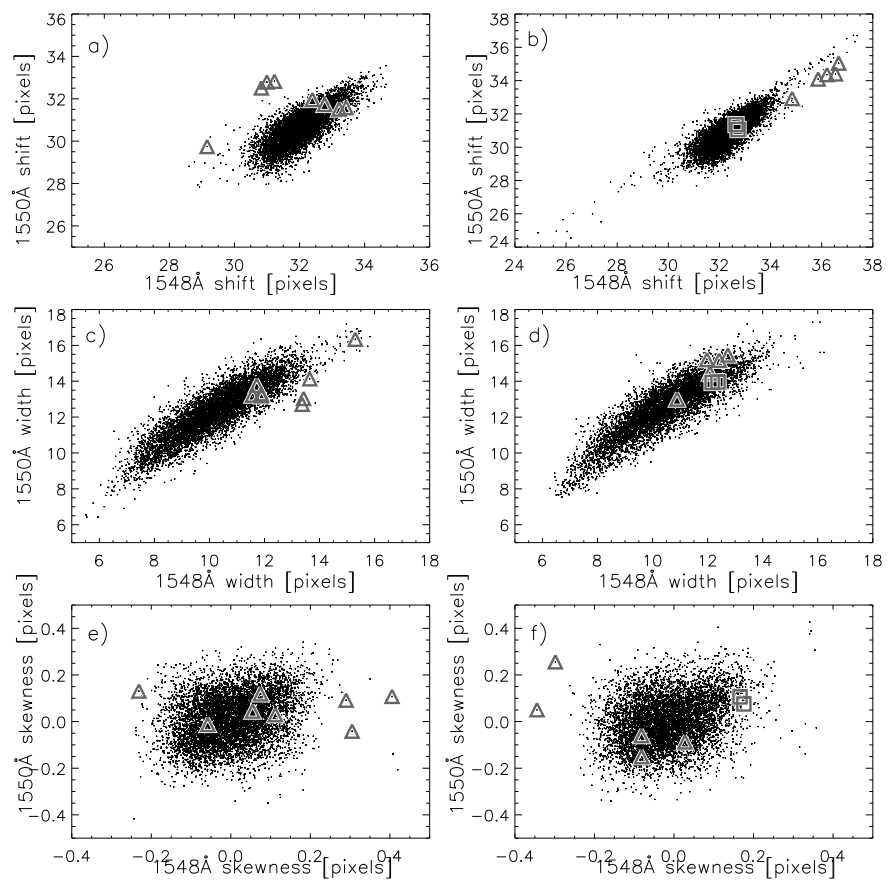

Fig. 3. Scatter plots of statistical parameters computed from the moments of the individual spectral profiles. The left-hand column of panels shows the data from raster 13:04-13:57 UT while the right-hand column shows the data from raster 13:58-14:53 UT. In each panel we show the moment value computed for the C IV $1550 \AA$ line as a function of the same moment value computed for the C IV $1548 \AA$ line. In panels a) and b) we show the Doppler shift, panels c) and d) the line width, and in panels e) and f) the skewness. In panels a), c), and e), the triangles indicate values from the profiles of Region 1 in Fig. 2 while in panels b),d), and f) triangles indicate values from profiles from Region 2 and squares from Region 3. Units are in SUMER spectral pixels.

combined with a visual inspection of the spatially nearby profiles. However, the use of the statistical indexes as a method for tracking asymmetric profiles was not completely successful. On the other hand, as we cannot find a combination of statistical parameters, such as the spectral width or the skewness of the two C IV spectral lines, as a clear indicator of the importance of asymmetric profiles, we cannot analyse any possible correlation between this indicator-parameter and other physical values deduced from the data. The study of the other data sets seen in Table 1 did not show any asymmetric profiles.

\subsection{Analysis of line blends}

We checked the possibility that the asymmetries in the profiles are caused by line blends. The C IV $1548 \AA$ is blended with Si I lines at $1547.36 \AA, 1547.45 \AA, 1548.72 \AA$ and an $\mathrm{O} \mathrm{V}$ line at $774.52 \AA$ (in the second order of the grating). The C IV $1550 \AA$ line is blended with Fe II $1550.26 \AA$ and Si I $1551.23 \AA$, where line identifications are from Curdt et al. (2001) and Peter (2000). The asymmetries in our profiles are caused by an enhanced spectral component in the blue wing of the C IV $1548 \AA$ while the corresponding spectral component is fainter or absent in the blue wing of the C IV $1550 \AA$ line. This is the case for all the profiles of Fig. 1 except panel $d$ which has a reversed C IV $1550 \AA$ profile.

Therefore, we will examine the possibility that a line in the blue wing of the C IV $1548 \AA$ is enhanced in intensity. In the 
spectral range of $1547.5 \AA$ to $1548 \AA$ we have the previously mentioned Si I lines. In a typical profile these lines are weaker than the C IV $1548 \AA$ line by a factor of 25 . Their intensity stays low relative to the $\mathrm{C}$ IV lines so that their effect is negligible. The other spectral blends, in the red wing of the C IV lines also have a weak intensity and cannot influence the asymmetries in the studied lines.

\section{Theoretical interpretation of asymmetric profiles}

\subsection{Importance of collisional and scattering terms in the formation of the CIV lines}

The C IV $1548 \AA$ and $1550 \AA$ spectral lines form a doublet as they have a common low energy level. The $1548 \AA$ line originates from the energy transition $1 \mathrm{~s}^{2} 2 \mathrm{~s}^{2} \mathrm{~S}_{1 / 2} \rightarrow 1 \mathrm{~s}^{2} 2 \mathrm{p}{ }^{2} \mathrm{P}_{3 / 2}$, which we refer to as the transition from ground level 1 to level 3. The $1550 \AA$ line originates from the atomic transition $1 \mathrm{~s}^{2} 2 \mathrm{~s}{ }^{2} \mathrm{~S}_{1 / 2} \rightarrow 1 \mathrm{~s}^{2} 2 \mathrm{p}{ }^{2} \mathrm{P}_{1 / 2}$, which we refer to as the transition from ground level 1 to level 2 . For this initial study we will assume that the C IV $1548 \AA$ and $1550 \AA$ lines are optically thin along the line of sight, which is the case for most of the solar disk (Dere \& Mason 1993). The specific intensities $I_{13}$ and $I_{12}$ of the C IV $1548 \AA$ and $1550 \AA$ lines are given respectively by the following equations (Kohl \& Withbroe 1982; Noci et al. 1987; Gontikakis et al. 2013):

$$
\begin{aligned}
& I_{13}(v)=h v_{13}\left(n_{\mathrm{i}} n_{\mathrm{e}} C_{13} \psi_{v}+n_{\mathrm{i}} B_{13} \bar{J}_{13}(v)\right) \frac{L}{4 \pi}, \\
& I_{12}(v)=h v_{12}\left(n_{\mathrm{i}} n_{\mathrm{e}} C_{12} \psi_{v}+n_{\mathrm{i}} B_{12} \bar{J}_{12}(v)\right) \frac{L}{4 \pi} .
\end{aligned}
$$

The right hand side of Eqs. (1) and (2) is composed by the sum of two terms. The first expresses the contribution of electron collisions and the second the contribution of scattering events. The collision terms are the product of the carbon ion population $n_{\mathrm{i}}$ times the electron density $n_{\mathrm{e}}$ and the collisional excitation rates $C_{13}$ or $C_{12}$. As the $1548 \AA$ and $1550 \AA$ are resonance lines, their low energy level is the ground level of the $\mathrm{C}^{3+}$ ions and the carbon ion population $n_{\mathrm{i}}$ replaces the population of the ions at ground level $n_{1}$. The carbon ion population $n_{\mathrm{i}}$ is calculated using the ionization equilibrium of CHIANTI v.7 and a carbon abundance from Feldman et al. (1992). In the scattering terms, $B_{13}$ and $B_{12}$ are the Einstein absorption coefficients and $\bar{J}_{13}(v), \bar{J}_{12}(v)$ are the radiative excitation rates (Noci et al. 1987). The quantities in Eqs. (1) and (2) are averaged over the thickness $L$, along the line of sight, of the emitting structure. The function $\psi_{v}$ is the emission profile of the collisional processes represented by a Gaussian function. The radiative excitation rates are expressed as

$$
\begin{aligned}
& \bar{J}_{13}(v)=\frac{\Omega}{4 \pi} \psi_{v} \int J_{13}\left(v^{\prime}\right) \phi_{v^{\prime}} \mathrm{d} v^{\prime}, \\
& \bar{J}_{12}(v)=\frac{\Omega}{4 \pi} \psi_{v} \int J_{12}\left(v^{\prime}\right) \phi_{v^{\prime}} \mathrm{d} v^{\prime},
\end{aligned}
$$

In Eqs. (3) and (4), $J_{13}\left(v^{\prime}\right)$ and $J_{12}\left(v^{\prime}\right)$ are the exciting radiation fields (Mihalas 1970; Rutten 2003; Hubeny \& Mihalas 2014), $\Omega$ is the solid angle corresponding to the region from where the exciting radiation originates. Finally, $\phi_{v}$ is the absorption profile. We consider that $\psi_{v}=\phi_{v}$ as we did not include coherent effects (Labrosse et al. 2006). In the following calculations, for most of the atomic parameters we used the CHIANTI atomic database (Dere et al. 1997; Landi et al. 2012).
Table 2. Physical parameters used for profile calculations.

\begin{tabular}{llll}
\hline \hline$n_{\mathrm{e} 1}\left[\mathrm{~cm}^{-3}\right]$ & $2 \times 10^{8}$ & $10^{9}$ & $5 \times 10^{9}$ \\
$n_{\mathrm{e} 2}\left[\mathrm{~cm}^{-3}\right]$ & $10^{9}$ & $5 \times 10^{9}$ & $10^{10}$ \\
$T_{1,2}[\mathrm{~K}]$ & $5 \times 10^{4}$ & $10^{5}$ & $5 \times 10^{5}$ \\
$\xi_{1,2}\left[\mathrm{~cm} \mathrm{~s}{ }^{-1}\right]$ & $10^{6}$ & $3 \times 10^{6}$ & $5 \times 10^{6}$ \\
$L_{1,2}[\mathrm{~cm}]$ & $10^{8}$ & $2 \times 10^{8}$ & $5 \times 10^{8}$ \\
$\Omega$ & $0.15 \pi$ & $0.2 \pi$ & $0.25 \pi$ \\
$\bar{J}_{13}\left[\mathrm{erg} \mathrm{s}^{-1} \mathrm{sr}^{-1} \mathrm{~cm}^{-2} \AA^{-1}\right]$ & $10^{4}$ & $4 \times 10^{4}$ & $8 \times 10^{4}$ \\
\hline
\end{tabular}

\subsection{Modelled profiles}

In this section we present our effort to simulate the observed profiles showed in Figs. 1a-c. We assume two plasma volumes, Volume 1 and Volume 2, which cannot be spatially distinguished by SUMER. Therefore the distance between them, projected on the plane of the sky is smaller than $1^{\prime \prime}$, the SUMER spatial pixel size. We also consider that they have different line of sight velocity components. Therefore, SUMER will record two spectral components in the C IV $1548 \AA$ and $1550 \AA$ lines, one for each volume. We call this effect a differential effect of velocity fields on resonant scattering. Both volumes are emitting photons due to collision excitation and to resonant scattering according to Eqs. (3), and (4). Due to the different plasma parameters in the two volumes, the fraction of collisional excitation over resonant scattering is not the same in Volumes 1 and 2. We suppose that resonant scattering is larger in the case of Volume 1.

This difference between the two volumes is parametrized with the lines ratio: the intensity ratio $R_{1}=I_{13}(1) / I_{12}(1)$ with the intensities $I_{13}(1), I_{12}(1)$ emitted by Volume 1 and the intensity ratio $R_{2}=I_{13}(2) / I_{12}(2)$ emitted by Volume 2 . In this case, $R_{1}>2$ and $R_{2}>2$ as resonant scattering affects both volumes. When resonant scattering is larger for Volume 1, we expect $R_{1}>R_{2}$.

Because of the differences in the line ratios, the spectral shape of the C IV $1548 \AA$ will be different from the shape of the C IV $1550 \AA$. We call this effect a differential velocity scattering. In this section we will examine the physical conditions in which such an effect on the spectral shapes is strong enough to be measurable.

The plasma parameters of Volumes 1 and 2 are the temperatures $T_{1}$ and $T_{2},[\mathrm{~K}]$ the electron densities $N_{\mathrm{e} 1}$ and $N_{\mathrm{e} 2},\left[\mathrm{~cm}^{-3}\right]$ the non-thermal velocities $\xi_{1}, \xi_{2}\left[\mathrm{~cm} \mathrm{~s}^{-1}\right]$, and the thickness of the volumes, $L_{1}$ and $L_{2}$, [cm], along the line of sight. Volume 1 is moving upward with a velocity $v_{1}=90 \mathrm{~km} \mathrm{~s}^{-1}$, while Volume 2 is at rest with $v_{2}=0 \mathrm{~km} \mathrm{~s}^{-1}$. The volumes are illuminated with incident intensities $\bar{J}_{13}(v)$ and $\bar{J}_{12}(v)$ for which we assume that $\bar{J}_{12}=\bar{J}_{13} / 2$. This means that the incident intensities are produced by collisional excitation. Moreover, we assume that the solid angle $\Omega$, from which the exciting radiation originates, is the same for the two volumes.

We selected three values for each parameter and computed artificial profiles in the C IV $1548 \AA$ and $1550 \AA$. The artificial profiles are composed of two spectral components with different Doppler shifts corresponding to $v_{1}$. The selected artificial profiles must be intense enough to be recorded with SUMER, and to have a measurable asymmetry. To quantify the asymmetry we calculate the intensity ratio of the two spectral components, $R_{1}=I_{13}(1) / I_{12}(1)$ and $R_{2}=I_{13}(2) / I_{12}(2)$ and we expect that $R_{1}$ is larger than $R_{2}$.

Table 2 shows the three values used for each of the plasma parameters. Plasma temperatures are selected to be half, equal 
or twice the maximum formation temperature of the $\mathrm{C}^{3+}$ ion, which is $10^{5} \mathrm{~K}$ (Mazzotta et al. 1998). Electron densities for Volume 1 are expected to be lower than those of Volume 2, as Volume 1 will be more affected by resonant scattering, which happens at relatively low electron densities (see Gontikakis et al. 2013). The non-thermal velocities $\xi_{1,2}$ are in the range $10^{6}$ to $5 \times 10^{6} \mathrm{~cm} \mathrm{~s}^{-1}$ that are relatively large values such as those observed in the asymmetric profiles. The incident intensity selected values are of $10^{4}$ to $8 \times 10^{4} \mathrm{erg} \mathrm{s}^{-1} \mathrm{sr}^{-1} \mathrm{~cm}^{-2} \AA^{-1}$ which correspond to plage emission (Brekke et al. 1991). In fact, the average peak intensity of the studied active region is $2 \times 10^{4} \mathrm{erg} \mathrm{s}^{-1} \mathrm{sr}^{-1} \mathrm{~cm}^{-2} \AA^{-1}$ while the peak intensity of the profile in Fig. 1a is $8 \times 10^{4} \mathrm{erg} \mathrm{s}^{-1} \mathrm{sr}^{-1} \mathrm{~cm}^{-2} \AA^{-1}$. The most difficult parameter to estimate is the solid angle $\Omega$. In Table 2 , the $\Omega$ values represent incident intensities originating from a bright structure, such as a bright point. Using the solid angle definition, $\Omega=A / d^{2}$ where $A$ is the intended bright point surface, illuminating Volumes 1,2 , and $d$ the distance from the microflare to the volumes. For $A=5\left({ }^{\prime \prime}\right)^{2}$ and $d=2.8^{\prime \prime}$ we would have $\Omega=0.2 \pi$. Therefore, we study the case where the asymmetric profiles are created through the illumination from a nearby bright point.

The combination of the parameters end up at 59049 profiles. We selected the profiles that are comparable with the observed asymmetric profiles. These are profiles where the C IV $1548 \AA$ peak intensities are higher than $10^{3} \mathrm{erg} \mathrm{s}^{-1} \mathrm{sr}^{-1} \mathrm{~cm}^{-2} \AA^{-1}$, where $R_{1}$ and $R_{2}$ are higher than 2.4 , and when $0.25<I_{13}(1) / I_{13}(2)<5$ so that the two spectral components have non-negligible intensities. We ended up with 376 profiles that satisfy these criteria.

Figure 4, shows four calculated spectral profiles with different shapes for each of the two lines as in the observed spectra. The thick line shows the C IV $1548 \AA$ profiles and the thin line those of C IV $1550 \AA$. The spectral component of Volume 1 is blueshifted while the one from Volume 2 is at rest. In panels a-c, $R_{1}=2.7$ while for panel d, $R_{1}=2.49$. Therefore, in all cases, the resonant scattering is important for the spectral component of Volume 1. For Volume 1, for all panels, the electron density is of $5 \times 10^{8} \mathrm{~cm}^{-3}$, and the temperature of $10^{5} \mathrm{~K}$. The low electron density is the cause of a weak collisional excitation component, so that resonant scattering becomes significant. The solid angle $\Omega$, for panels a to c, equals $0.25 \pi$ while for panel $\mathrm{d}$ it is reduced to $0.15 \pi$, which explains the lower $R_{1}$ value there. On the other hand, the Volume 1 spectral component is of higher intensity for panel c, because the length $L_{1}$ equals $2 \times 10^{8} \mathrm{~cm}$ for panels a, $\mathrm{b}$ and d, while for panel c, $L_{1}=5 \times 10^{8} \mathrm{~cm}$. The non-thermal velocity $\xi_{1}=10^{6} \mathrm{~cm} \mathrm{~s}^{-1}$ for all cases.

The spectral component of Volume 2 is marginally affected by resonant scattering for panels a-c as $R_{2} \simeq 2$ except for panel d where $R_{2} \simeq 2.3$. The electron density, in Volume 2 , for panel a is $5 \times 10^{9} \mathrm{~cm}^{-3}$, for panels $\mathrm{b}$ and $\mathrm{c}$ it equals $10^{10} \mathrm{~cm}^{-3}$, while for panel d it is $10^{9} \mathrm{~cm}^{-3}$. The temperature of Volume 2, for the four panels is $1.5 \times 10^{5} \mathrm{~K}, 3 \times 10^{5} \mathrm{~K}, 1.5 \times 10^{5} \mathrm{~K}$, and $10^{5} \mathrm{~K}$, respectively. Therefore the enhanced $R_{2}$ value in panel d is caused by the reduced electron density causing a relatively more important resonant scattering component, even if the solid angle is smaller in this case as we saw previously. The nonthermal velocity for Volume 2 is $3 \times 10^{6} \mathrm{~cm} \mathrm{~s}^{-1}, 3 \times 10^{6} \mathrm{~cm} \mathrm{~s}^{-1}$, $6 \times 10^{6} \mathrm{~cm} \mathrm{~s}^{-1}$, and $3 \times 10^{6} \mathrm{~cm} \mathrm{~s}^{-1}$ for panels a to d respectively. The Volume 2 spectral component intensity is higher in panel $\mathrm{c}$ than in panel $\mathrm{b}$ by a factor of $\simeq 12$ even if the electron density is the same for both cases. The reason is that the temperature is lower in panel $\mathrm{c}$ by a factor of two which enhances the $\mathrm{C}^{3+}$ ion concentration by a factor of 23 as the temperature is now
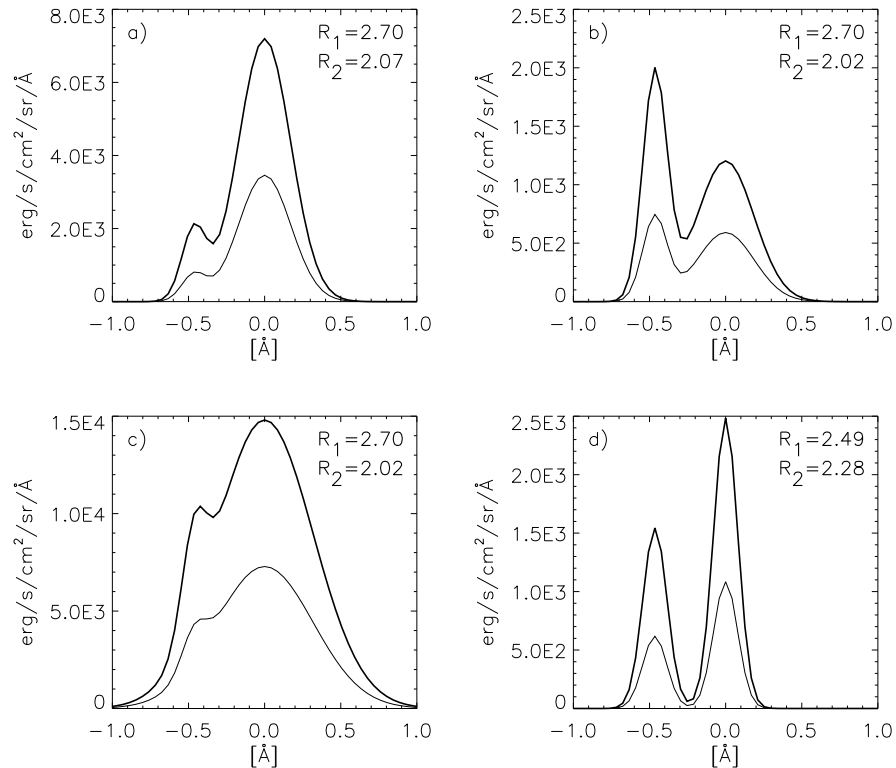

Fig. 4. Computed spectral profiles of C IV $1548 \AA$ (thick line) and C IV $1550 \AA$ (thin line). The blueshifted spectral component corresponds to Volume 1 and Volume 2 corresponds to the central component with zero Doppler shift. All profiles are computed with an incident intensity of $8 \times 10^{4} \mathrm{erg} \mathrm{s}^{-1} \mathrm{~cm}^{-2} \mathrm{sr}^{-1} \AA^{-1}$, and $\Omega=0.25 \pi$ for panels a) to $\mathbf{c}$ ) and $0.2 \pi$ for panel d). The spectral components intensity ratios $R_{1}$ and $R_{2}$ are indicated in the panels. For panels a) to c), $R_{1} / R_{2} \simeq 1.3$ and for panel d) $R_{1} / R_{2} \simeq 1.1$. In all cases, $n_{e 1}=5 \times 10^{8} \mathrm{~cm}^{-3}, T_{1}=10^{5} \mathrm{~K}$. $n_{e 2}=5 \times 10^{9} \mathrm{~cm}^{-3}$ (panels a) and d)) and $n_{e 2}=5 \times 10^{10} \mathrm{~cm}^{-3}$ (panels b) and c)).

closer to the maximum ion formation temperature of $10^{5} \mathrm{~K}$. On the other hand, the non-thermal velocity in panel $\mathrm{c}$ is twice that of panel $b$ which reduces the intensity by a factor close to two. Therefore the variations in temperature and non-thermal velocity are the parameters which modify the intensity in panels $b$ and $c$.

In all panels except $b$ the Volume 1 component has a weaker intensity than the Volume 2 one. The case of panel $\mathrm{c}$ has the highest intensities and highest $\xi_{2}$ values. For the spectral component ratios the relation $R_{1} / R_{2}>1$ holds. The $R_{1} / R_{2}$ becomes higher for lowest electron densities in Volume 1. $R_{1} / R_{2}$ is related to $T_{1}$ and $T_{2}$ through the collision excitation coefficient.

\subsubsection{Model applied for the Si Iv $1393.757 \AA$ and $1402.772 \AA$ lines}

We repeated the analysis in Sect. 5.2 for the Si IV $1393.757 \AA$ and $1402.772 \AA$ line doublet (Landi et al. 2012). The $\mathrm{Si}^{3+}$ ion has a formation temperature of $\simeq 80000 \mathrm{~K}$, (Landi et al. 2012), close to the formation temperature of the $\mathrm{C}^{3+}$ ion. The oscillation strengths of Si IV $1393 \AA$ and $1402 \AA$ are $f_{13}=0.513$ and $f_{12}=0.255$ respectively (Kramida et al. 2014). This is higher than the corresponding oscillation strengths of the C IV lines by a factor of about 2.6. Moreover, the collisional excitation strengths of the Si IV lines are also higher by a factor of $\simeq 2$ relative to those of the $\mathrm{C}$ IV lines (Landi et al. 2012). Therefore the two terms of Eqs. (1) and (2) have a similar relative strength for the two doublets. The recorded intensities of the two doublets are similar according to SUMER measurements (Curdt et al. 2001). We ran the model with the same parameters as in Table 2, replacing the $10^{5} \mathrm{~K}$ temperature with $80000 \mathrm{~K}$. We found similar asymmetric profiles as in Fig. 4. Therefore we expect also to find asymmetric profiles in explosive events recorded with the 
Interface Region Imaging Spectrograph (IRIS; De Pontieu et al. 2014) which observes the Si IV lines with high spectral and spatial accuracy.

\subsection{The reversed profiles}

The most difficult profiles to interpret are the ones similar to that in Fig. 1d where the C IV $1550 \AA$ line is self-reversed while the C IV $1548 \AA$ line presents a single component. In Sect. 3, we presented the possibility that the two spectral peaks of the C IV $1550 \AA$ correspond to two spectral components with different Doppler shifts which are not apparent in the C IV $1548 \AA$ line. We also said that the profile of C IV $1550 \AA$ could be reversed due to optical thickness. As the optical thickness of C IV $1548 \AA$ is twice that of C IV $1550 \AA$, one should expect that the C IV $1548 \AA$ would also be reversed, but this is not the case. We propose the following combination of the two emitting volumes: Volume 1 has a high opacity and consequently emits reversed profiles, where $I_{13}(1)=I_{12}(1)$; on the contrary in Volume 2, the diffusion scattering dominates and $I_{13}(2)=4 I_{12}(2)$ and where the profiles are not reversed. The sum of the profiles from the two volumes could produce a selfreversed C IV $1550 \AA$ line and a C IV $1548 \AA$ where only one spectral profile is apparent.

\subsection{Possible importance of linear polarisation}

SUMER is sensitive to linearly polarised light, because of the large incident angle on SUMER's mirror. Its sensitivity has been measured before flight, and presented in Hassler et al. (1997). In Hassler et al. (1997), for a fully linearly polarised incident beam ( $p=1)$, the maximum response of the instrument, corresponding to $p \simeq 0.6$ is found for wavelengths in the range of $1540 \AA$ to $1550 \AA$ (see their Fig. 6).

Light emitted in the C IV $1548 \AA$ line, when resonance scattering is dominant, may be linearly polarised if the incident radiation is not isotropic (Stenflo \& Stenholm 1976; Trujillo Bueno et al. 2005). On the other hand, the C IV $1550 \AA$, is not producing linearly polarised light during scattering. This means that a fraction of the measured profile asymmetries, in Fig. 1, may be due to linear polarisation of the C IV $1548 \AA$ photons, when resonant scattering is dominant, and as long as the polarisation plane is nearly parallel to SUMER's slit.

This effect was measured for the doublet emitted by $\mathrm{O}^{5+}$, which is also a lithium-like ion similar to $\mathrm{C}^{3+}$. Raouafi et al. (1999), measured the linear polarisation of the O VI $1032 \AA$ line, caused by resonance scattering, in contrast to the non-affected O VI $1037 \AA$ line, at the south limb. There, the rotation maneuver of the SOHO satellite provided measurements with different orientations of the SUMER slit, giving a quantitative measurement of $p$, which becomes maximum at the solar limb. The polarisation properties are the same for the C IV lines. Stenflo \& Stenholm (1976), presents calculations of the polarisation for the spectral line Si IV $1393 \AA$ which has the same ability to polarise light as the C IV $1548 \AA$ and O VI $1032 \AA$. Moreover, these three spectral lines have the same polarisation factor $E_{1}=0.5$ as deduced from Dumont et al. (1973). In Stenflo \& Stenholm (1976), an optically thin transition region, at the limb, will produce a $p=0.17$ for the Si IV line. Moreover, we expect a maximum polarisation measurement at the limb. We expect that the fraction of linear polarisation will be lower than 0.17 in our observed profiles. Such a calculation will be the subject of a future project.

\section{Discussion and conclusions}

We studied few cases, where the C IV $1548 \AA$ and $1550 \AA$ spectral lines, at specific locations, observed at the same time, present different spectral shapes (Fig. 1).

These profiles, which we call asymmetric profiles, were found at three different locations in the studied active region (Fig. 2). This means that this effect is not common. We did not find any asymmetry in the quiet Sun profiles. However, the quiet Sun data had a small f.o.v. of only one SUMER slit position. One should study quiet Sun data with a larger f.o.v. to search for possible asymmetric profiles.

Searching such events is difficult not only because of their sparsity. Asymmetric profiles can be confused with profiles affected by statistical noise. However, in the scatter plots of Fig. 3, the asymmetric profiles can be identified as they lay outside the main point distributions.

Such a variation in profiles of the $C$ IV lines is excluded if the lines are formed only through collisional excitations. The lines are emitted from the same ion so that even complex plasma motions should produce the same Doppler shifts in the line shapes.

The observed profiles may be explained if two mechanisms participated in their formation: resonant scattering and collisional excitation. The scenario implies two plasma volumes, observed within a single SUMER spatial pixel, moving with different velocities, projected towards the observer. If the resonant scattering is dominant only in one plasma volume, then their combined emission can form asymmetric profiles such as in the observations.

In Sect. 5, the calculated profiles showed that this mechanism can produce asymmetric profiles for plasma parameters that are typical in the solar transition region. Figure 4 shows that the plasma volume dominated by resonant scattering, has a smaller electron density which is the reason for the weak collisional term. The analysis of such observed profiles can allow the diagnostic of the plasma parameters in the different spectral components.

In the literature there are cases where C IV $1548 \AA$ and $1550 \AA$ lines profiles present asymmetries or different Doppler shifts as mentioned in our introduction. We believe that at least in some cases, differential effect of velocity fields on resonant scattering may explain these observations.

The observed profiles may be affected by linearly polarised light since SUMER is sensitive to it. A preliminary computation shows that it may be of a few percent as in the case measured by Raouafi et al. (1999). This issue remains to be addressed in a future work. Finally, we should point out that even if C IV $1548 \AA$ has a small optical depth, a more detailed analysis should also take into account the effect of opacity.

Such an analysis should include more relevant observations since SUMER provided several data points which recorded the C IV $1548 \AA, 1550 \AA$ doublet where such asymmetric profiles could be found.

The search for asymmetric profiles with variations from one line to the second one in a doublet seems to be promising for spectral observations above the limb. Contrary to disk observations, the lines are only emitted in low electron density altitudes. Therefore resonant scattering is often dominant above the limb. The search for asymmetric profiles in off-limb events, such as those described in Tavabi et al. (2015) where Si IV lines are recorded with IRIS, would allow for a new and more complete diagnostic of coronal plasmas. 
Acknowledgements. We would like to thank L. Teriaca and M. Madjarska for interesting discussions, and P. Judge for reading a first version of our manuscript. We would also like to thank our anonymous referee and the editor for interesting remarks that enhanced the scientific quality of our work. CHIANTI is a collaborative project involving George Mason University, the University of Michigan (USA) and the University of Cambridge (UK). The SUMER project is financially supported by DLR, CNES, NASA and the ESA PRODEX Programme (Swiss contribution). SUMER is part of the ESA/NASA SOHO mission. CG acknowledges financial aid from the grant 200/790 of the research committee of the Academy of Athens.

\section{References}

Arnaud, M., \& Rothenflug, R. 1985, A\&AS, 60, 425

Brekke, P., Hassler, D. M., \& Wilhelm, K. 1997, Sol. Phys., 175, 349

Brekke, P., Kjeldseth-Moe, O., Bartoe, J.-D. F., \& Brueckner, G. E. 1991, ApJS, 75,1337

Curdt, W., \& Tian, H. 2011, A\&A, 532, L9

Curdt, W., Brekke, P., Feldman, U., et al. 2001, A\&A, 375, 59

De Pontieu, B., Title, A. M., Lemen, J. R., et al. 2014, Sol. Phys., 289, 2733

Dere, K. P., \& Mason, H. E. 1993, Sol. Phys., 144, 217

Dere, K. P., Bartoe, J.-D. F., \& Brueckner, G. E. 1989, Sol. Phys., 123, 41

Dere, K. P., Landi, E., Mason, H. E., Monsignori Fossi, B. C., \& Young, P. R. 1997, A\&AS, 125

Dumont, S., Pecker, J.-C., \& Omont, A. 1973, Sol. Phys., 28, 271

Feldman, U., Mandelbaum, P., Seely, J. F., Doschek, G. A., \& Gursky, H. 1992, ApJS, 81, 387

Gontikakis, C., Winebarger, A. R., \& Patsourakos, S. 2013, A\&A, 550, A16

Hassler, D. M., Lemaire, P., \& Longval, Y. 1997, Appl. Opt., 36, 353

Hubeny, I., \& Mihalas, D. 2014, Theory of Stellar Atmospheres (Princeton: Princeton University Press)
Innes, D. E., Guo, L.-J., Huang, Y.-M., \& Bhattacharjee, A. 2015, ApJ, 813, 86

Judge, P. G., \& Pietarila, A. 2004, ApJ, 606, 1258

Kohl, J. L., \& Withbroe, G. L. 1982, ApJ, 256, 263

Kramida, A., Ralchenko, Y., \& Reader, J. 2014, in APS Division of Atomic, Molecular and Optical Physics Meeting Abstracts

Labrosse, N., Li, X \& \&i, B. 2006, A\&A, 455, 719

Landi, E., Del Zanna, G., Young, P. R., Dere, K. P., \& Mason, H. E. 2012, ApJ, 744, 99

Lites, B. W., \& Cook, J. W. 1979, ApJ, 228, 598

Mariska, J. T. 1992, The solar transition region (New York: Cambridge University Press)

Mazzotta, P., Mazzitelli, G., Colafrancesco, S., \& Vittorio, N. 1998, A\&AS, 133, 403

Mihalas, D. 1970, Stellar atmospheres (San Francisco: Freeman)

Noci, G., Kohl, J. L., \& Withbroe, G. L. 1987, ApJ, 315, 706

Peter, H. 2000, A\&A, 360, 761

Raouafi, N.-E., Lemaire, P., \& Sahal-Bréchot, S. 1999, A\&A, 345, 999

Rutten, R. J. 2003, in Stellar Atmosphere Modeling, eds. I. Hubeny, D. Mihalas, \& K. Werner, ASP Conf. Ser., 288, 99

Stenflo, J. O., \& Stenholm, L. 1976, A\&A, 46, 69

Tavabi, E., Koutchmy, S., \& Golub, L. 2015, Sol. Phys., 290, 2871

Teriaca, L., Banerjee, D., \& Doyle, J. G. 1999, A\&A, 349, 636

Trujillo Bueno, J., Landi Degl'Innocenti, E., Casini, R., \& Martínez Pillet, V. 2005, in 39TH ESLAB Symposium on Trends in Space Science and Cosmic Vision 2020, eds. F. Favata, J. Sanz-Forcada, A. Giménez, \& B. Battrick, ESA SP, 588, 203

Wilhelm, K., Curdt, W., Marsch, E., et al. 1995, Sol. Phys., 162, 189

Winebarger, A. R., Warren, H., van Ballegooijen, A., DeLuca, E. E., \& Golub, L. 2002, ApJ, 567, L89

Wood, B. E., Linsky, J. L., \& Ayres, T. R. 1997, ApJ, 478, 745 